\title{
Fotoprotetor: Desenvolvimento, Estudo de Estabilidade Preliminar e Avaliação in vitro do Fator de Proteção Solar (FPS).
}

\author{
Sunscreen: Development, Preliminary Stability Tests \\ and Evaluation In Vitro of Sun Protection Factor (SPF).
}

Recebido em: 13/08/2016 Aceito em: $12 / 03 / 2017$
Tamara GONÇALVES ${ }^{1}$; Aline Cordeiro de ALMEIDA²; Bianca Oliveira LOUCHARD ${ }^{2}$

${ }^{1}$ Laboratório de Cosmetologia, Departamento de Farmácia, Faculdade de Farmácia, Odontologia e Enfermagem, Universidade Federal do Ceará. Rua Cap. Francisco Pedro, 1210. Rodolfo Teófilo, CEP 60430-370. Fortaleza, CE, Brasil. 2 Faculdade de Farmácia, Odontologia e Enfermagem, Universidade Federal do Ceará. Rua Cap. Francisco Pedro, 1210. Rodolfo Teófilo, CEP 60430-370.Fortaleza, CE, Brasil.E-mail: tamara.ufc@gmail.com

\begin{abstract}
Sunscreen use is the most common photoprotection alternative used by the population, and so these products should offer improved protection with broad - spectrum, UVA and UVB protection. The aim of the present study was to develop sunscreens emulsions and to evaluate their preliminary stability and in vitro photoprotective efficacy. Five formulations were prepared by phase inversion using differences concentrations of Non-ionic self-emulsifying base, Non-ionic surfactant and viscosity agent. The in vitro Sun Protect Factor (SPF) was determined by spectofotometer method and the formulations were stored at 25 e $37^{\circ} \mathrm{C}$ by fifteen days. Preliminary stability tests was carried out in triplicate with evaluation by macroscopic analysis, $\mathrm{pH}$ testing, centrifugation and viscosity assessment. The results demonstrated that two formulations (F4 and F5) were stable, but the formulation F4 presented high viscosity. In summary, the formulation F5 (SPF 24.32 \pm 0.025 ) was stable and presented the most agreeable sensory properties.
\end{abstract}

Keywords: Sunscreen, Stability, Cetearyl Alcohol.

\section{RESUMO}

O uso de protetor solar é uma das medidas de fotoproteção mais utilizadas pela população, portanto esses produtos devem oferecer eficácia na proteção contra as radiações solares além de garantir segurança e qualidade da formulação desenvolvida. Os autores tiveram como objetivo desenvolver, avaliar a estabilidade preliminar e determinar o FPS in vitro de emulsões fotoprotetoras. Foram preparadas cinco formulações, pelo método de inversão de fases, utilizando diferentes concentrações de base autoemulsionante não iônica, tensoativo não iônico e agente de consistência. O Fator de Proteção Solar (FPS) foi determinado in vitro por método espectrofotométrico, e as formulações armazenadas a 25 e $37^{\circ} \mathrm{C}$ durante quinze dias. Foram realizados os ensaios de centrifugação, análise visual, medidas de pH e viscosidade. Os resultados demonstraram que duas formulações (F4 e F5), apresentaram estabilidade, porém a formulação F4 apresentou alta viscosidade. Em conclusão, a formulação F5 (FPS 24.32 \pm 0.025 ) apresentou estabilidade e adequadas propriedades sensoriais.

Palavras-chave: Fotoprotetor, Estabilidade, Álcool cetoestearilico. 


\section{INTRODUÇÃO}

A pele está constantemente exposta à radiação ultra violeta (UV), acreditando-se ser este o maior mediador exógeno de danos (1). Os fotoprotetores ou protetores solares são preparações cosméticas de defesa frente aos efeitos danosos da radiação UV. Eles podem ser compostos de filtros inorgânicos e orgânicos, e preparados na forma de loções hidroalcoólicas, óleos, géis, emulsões, bastões, aerossóis, entre outras (2).

As emulsões são formas cosméticas populares, preferidas por formuladores e consumidores, porque se espalham com facilidade na pele, além de apresentarem maior proteção $(2,3)$. Emulsão é um sistema termodinamicamente instável resultante da mistura de duas fases imiscíveis entre si, estabilizadas por um agente emulsificante (4). Com o passar do tempo, as emulsões tendem a retornar ao seu estado fundamental, ou seja, tendem a separação de fases. Os processos que precedem a separação de fases podem ser identificados como sinais de instabilidade e observados na preparação na forma de cremeação, sedimentação, alterações organolépticas e físico-químicas. Estudos de estabilidade visam predizer o tempo em que as preparações mantem inalteradas as características apresentadas após o preparo (5).

O estudo da estabilidade de produtos cosméticos fornece informações que indicam o grau de estabilidade de um produto nas variadas condições a que possa estar sujeito desde sua fabricação até o término do prazo de validade (5).

Diante do exposto, os autores tiveram como objetivo a partir de uma formulação comercial com problemas de estabilidade, desenvolver, avaliar a estabilidade preliminar, e determinar o FPS in vitro de uma nova emulsão fotoprotetora.

\section{MATERIAL E MÉTODOS}

Componentes das Formulações. Foram preparadas 5 formulações de emulsões O/A(F1, F2, F3, F4 e F5), como mostra a Tabela 1. A formulação F1 correspondeu a uma formulação comercial manipulada e comercializada pela Farmácia Escola da Universidade Federal do Ceará (UFC), que vinha apresentando sinais de instabilidade. Desta forma, tomando como base a formulação F1, foram desenvolvidas, sequencialmente, formulações alterando a composição quali e quantitativa de matérias primas a fim de se conseguir uma formulação que apresentasse estabilidade adequada. A técnica de preparo das emulsões foi pelo processo de inversão de fases. As fases aquosa e oleosa foram aquecidas separadamente (70$75^{\circ} \mathrm{C}$ ), e em seguida a fase aquosa foi vertida lentamente sob a fase oleosa, sob agitação mecânica constante (Fisatom ${ }^{\circledR} \bmod$. 713A), mantendo aquecimento por 15 minutos. Após esse período a dispersão de dióxido de titânio foi adicionada sob agitação, e a preparação foi resfriada a $25^{\circ} \mathrm{C}$, mantendo agitação constante.

Tabela 1. Composição quantitativa (\% p/p) das matérias-primas utilizadas em cada formulação avaliada

\begin{tabular}{|c|c|c|c|c|c|}
\hline Componentes (INCI) & F1 (\%p/p) & F2 (\%p/p) & F3 (\%p/p) & F4 (\%p/p) & F5 (\%p/p) \\
\hline Cetearyl Alcohol (1) & * & * & * & 4,5 & 4,0 \\
\hline Cetearyl Alcohol, Ceteareth-20 (1) & 5,6 & 4,5 & 4,5 & * & * \\
\hline Ceteareth - 20 (1) & 1,8 & 1,5 & 1,5 & 1,5 & 2,0 \\
\hline VP/Eicosene Copolymer ${ }^{(1)}$ & 2,8 & 2,8 & 2,8 & 2,8 & 2,8 \\
\hline Ethylhexyl Methoxycinnamate ${ }^{(1)}$ & 8,9 & 8,0 & 8,0 & 8,0 & 8,0 \\
\hline Benzophenone-3 ${ }^{(1)}$ & 3,7 & 3,0 & 3,0 & 3,0 & 3,0 \\
\hline Ethylhexyl Salicylate ${ }^{(1)}$ & 1,8 & 3,0 & 3,0 & 3,0 & 3,0 \\
\hline $\mathrm{BHT}^{(1)}$ & 0,1 & 0,1 & 0,1 & 0,1 & 0,1 \\
\hline Titanium dioxide ${ }^{(3)}$ & 2,8 & 3,0 & * & 3,0 & 3,0 \\
\hline Methyldibromo Glutaronitrile, Phenoxyethanol & 0,09 & 0,09 & 0,09 & 0,09 & 0,09 \\
\hline Dissodium EDTA & 0,1 & 0,1 & 0,1 & 0,1 & 0,1 \\
\hline Propylene glycol(2) & 4,5 & 5,0 & 5,0 & 5,0 & 5,0 \\
\hline Disttiled water ${ }^{(2)}$ & q.s.p & q.s.p & q.s.p & q.s.p & q.s.p \\
\hline
\end{tabular}

* Ausência da matéria-prima na formulação; ${ }^{(1)}$ Componentes da Fase Oleosa; ${ }^{(2)}$ Componentes da Fase Aquosa; ${ }^{(3)}$ Componentes da dispersão 
Estudo de Estabilidade Preliminar. As preparações foram envasadas em frascos transparentes e armazenadas em temperatura controlada e ao abrigo da luz: temperatura ambiente (TA), $25 \pm 2^{\circ} \mathrm{C}$; e estufa (Labtrade ${ }^{\circledR}$ Mod. EB100), $37 \pm 2^{\circ} \mathrm{C}$, por um período de 15 dias, para avaliação da estabilidade preliminar (5). As formulações foram analisadas quanto ao aspecto físico, características organolépticas (cor e odor), $\mathrm{pH}$, viscosidade e teste de centrifugação. As análises foram realizadas no $1^{\circ}$ e $15^{\circ}$ dias após preparo.

Avaliação organoléptica. Foram observadas alterações do tipo cor, odor, separação de fases e homogeneidade da preparação. Para a análise dos resultados, foram utilizados os critérios de classificação adotados por Larentes, (2009): Normal: sem alteração; levemente separado ou levemente precipitado; separado ou precipitado (6).

Teste de centrifugação. As formulações foram colocadas em tubos cônicos para centrifugação (Evlab ${ }^{\circledR}$ mod. EV:04) e submetidas a 3000 rotações por minuto (rpm) durante 30 minutos à temperatura ambiente. Ao final do ensaio qualquer sinal de separação de fases ou precipitação indica a necessidade de reformulação. Se aprovado nesse teste, o produto segue para as demais avaliações (5).

Determinação de pH. As amostras foram submetidas à avaliação de $\mathrm{pH}$, através da utilização de um potenciômetro (Gehaka, mod. PG 2000). Após a calibração, foi feita a leitura do $\mathrm{pH}$ introduzindo-se o eletrodo diretamente na amostra diluída na concentração de $10 \%$ em água destilada. A análise foi realizada em triplicata.

Viscosidade. A viscosidade foi determinada utilizando viscosímetro rotacional (Haake ${ }^{\circledR}$ mod. visco Tester 61), empregando o spindle de número 3 (L3). O spindle foi incorporado à amostra de modo a evitar a formação de bolhas de ar em contato com a superfície do mesmo. $\mathrm{O}$ valor da viscosidade foi expresso em centipoise (cP) com torque próximo a 50\%.

Determinação do Fator de Proteção Solar. O Fator de Proteção Solar (FPS) foi determinado segundo metodologia in vitro proposta por Mansur e cols (1986), com algumas adaptações (7). As absorbâncias das amostras foram mensuradas utilizando um espectrofotômetro UV/VIS (Genesys 10V). As formulações foram diluídas com etanol $95^{\circ} \mathrm{GL}$ até obtenção da concentração final de $0,2 \mu \mathrm{L} / \mathrm{mL}$, e filtradas com auxílio de uma seringa, em membrana de $0,22 \mu \mathrm{m}$ de poro para obtenção de uma solução límpida. Esta etapa da filtração foi necessária para retirada de partículas suspensas de dióxido de ti- tânio, que poderiam opacificar a amostra, e interferir na análise espectrofotométrica. As amostras foram preparadas em triplicata. As absorbâncias das soluções foram determinadas na faixa de 290 a $320 \mathrm{~nm}$, com intervalos de $5 \mathrm{~nm}$, utilizando-se etanol como branco. Para cada comprimento de onda foram realizadas três determinações. O cálculo do FPS foi realizado de acordo com a Equação 1 (7).

$$
F P S=F C \sum_{290}^{320} E E(\lambda) \mathrm{I}(\lambda) \cdot \operatorname{abs}(\lambda)
$$

Onde:

$\mathrm{Fc}=$ fator de correção (igual a 10); $\mathrm{EE}(\lambda)=$ efeito eritematogênico da radiação solar em cada comprimento de onda $\lambda$; I $(\lambda)=$ intensidade da luz solar no comprimento de onda; $\operatorname{Abs}(\lambda)=$ leitura espectrofotométrica da absorbância da amostra em cada comprimento de onda.

Os resultados das leituras foram multiplicados pelo efeito eritematogênico da radiação e pela intensidade do sol, específicos de cada comprimento de onda (Tabela 2).

Tabela 2. Relação entre o efeito eritematogênico e a intensidade da radiação em cada comprimento de onda (Mansur e cols, 1986)

\begin{tabular}{|c|c|}
\hline Comprimento de onda $\lambda(\mathrm{nm})$ & $\mathrm{EE}(\lambda) \times \mathrm{I}(\lambda)$ \\
\hline 290 & 0,0150 \\
\hline 295 & 0,0817 \\
300 & 0,2874 \\
\hline 305 & 0,3278 \\
\hline 310 & 0,1864 \\
315 & 0,0839 \\
320 & 0,0180 \\
\hline
\end{tabular}

Análise estatística. A análise dos dados foi realizada através da determinação da média e desvio padrão, além de análise de variância ANOVA seguida pelo teste de Tukey $(p<0,05)$, quando adequado.

\section{RESULTADOS E DISCUSSÃO}

Aspecto físico e Avaliações Organolépticas. As 5 formulações (F1, F2, F3, F4 e F5) armazenadas a $25 \pm 2^{\circ} \mathrm{C}$ durante 15 dias, não apresentaram alterações em relação ao aspecto físico ou as características organolépticas (cor e odor) por análise visual a olho nu contra fundo branco (5). Quando armazenadas à $37 \pm 2^{\circ} \mathrm{C}$, foi observada separação de fases na formu- 
lação F1 e formação de precipitados nas formulações F2 e F3. As formulações F4 e F5 foram as únicas que não sofreram alterações com o aumento da temperatura $\left(37 \pm 2^{\circ} \mathrm{C}\right)$.

A formulação $\mathrm{F} 1$, quando armazenada à $37 \pm 2^{\circ} \mathrm{C}$, apresentou cremagem no décimo quinto dia $\left(15^{\circ} \mathrm{dia}\right) \mathrm{de}$ análise. Isso pode ocorrer como consequência do agrupamento das gotículas oleosas da fase interna, devido às diferenças de densidade entre as fases que constituem o sistema $(8,9)$. Esse efeito, quando ocorre de forma isolada, nem sempre é considerado sinal de instabilidade da preparação, uma vez que é um processo reversível, onde a uniformidade da dispersão pode ser restabelecida mediante simples agitação da emulsão, pois as gotículas ainda estão circundadas por uma camada protetora de agente emulsionante, porém, essa prática não é atraente para os consumidores, uma vez que não há uniformidade da formulação. Para aumentar a estabilidade dessas formulações pode-se reduzir o tamanho das gotículas através de processos mecânicos de homogeneização ou fazer uso de agentes emulsionantes, os quais facilitam o processo de emulsificação gerando produtos com gotículas de tamanho menor, que favorecem a estabilidade do sistema. Outra possibilidade é adicionar um agente espessante para aumentar a viscosidade da fase contínua/externa (10).

No presente trabalho, foi observado que a cremagem sofrida pela amostra F1, foi, possivelmente, resultado de uma alta proporção de compostos etoxilados que favorece a liquefação da emulsão. A base autoemulsionante e agente de consistência cosmowax J (Cetearyl Alcohol, Ceteareth-20), que já possui em sua composição álcool cetoestearílico etoxilado, foi usada na amostra F1 na proporção de 5,6\% p/p, e, uma vez, associada a 1,8\% de álcool cetoestearílico etoxilado (Ceteareth - 20), pode ter desestabilizado o sistema por excesso de agente surfactante etoxilado, favorecendo a liquefação da emulsão quando submetida à alta temperatura $\left(37 \pm 2^{\circ} \mathrm{C}\right)$.

Para confirmar essa hipótese, foi desenvolvida a formulação F2 com proporções de cosmowax J e álcool cetoestearílico etoxilado reduzidos para 4,5 e 1,5\%, respectivamente. Após o período de 15 dias, sob armazenamento à $37 \pm 2^{\circ} \mathrm{C}$, foi observada a formação de precipitado. Sabe-se que dispersão de dióxido de titânio, em alguns casos, pode ser a causa da precipitação observada em formulações de fotoprotetores (11), por essa razão, foi desenvolvida a formulação $\mathrm{F} 3$ com a mesma composição quali e quantitativa de F2, com exceção do dióxido de titânio. Após 15 dias de armazenamento à
$37 \pm 2^{\circ} \mathrm{C}$, a formulação $\mathrm{F} 3$ também apresentou formação de precipitados, permitindo inferir que essa instabilidade não está associada ao dióxido de titânio, e sim, à quantidade insuficiente de agente espessante, visto que ao espessar a fase continua ou mesmo a fase interna da emulsão processos de sedimentação ou cremeação podem ser evitados. Desta forma, para o desenvolvimento da formulação F4, substituiu-se a base autoemulsionante cosmowax J pelo, agente espessante de fase oleosa, o álcool cetoestearílico, mantendo a mesma proporção de utilização da base $(4,5 \%$ p/p).

A formulação $\mathrm{F} 4$ se mostrou estável, tanto à temperatura ambiente quanto a $37 \pm 2^{\circ} \mathrm{C}$, porém com uma viscosidade muito superior as emulsões fotoprotetoras convencionais, característica que não a tornava atraente para o consumidor final. Com o intuito de manter a estabilidade do produto e melhorar o sensorial, foi desenvolvida a formulação F5, diminuindo a concentração do agente espessante para $4,0 \%$, e aumentando a quantidade do emulsionante etoxilado para $2,0 \%$. Como resultado, obteve-se uma formulação mais fluida que a anterior, e estável a todas as condições de armazenamento submetidas. Com esse estudo preliminar, pode-se concluir que a proporção e a seleção dos componentes, entre eles, emulsionantes e espessantes, é um fator crítico para obter a estabilidade de uma emulsão, como também para lograr a viscosidade ideal.

Nenhuma das formulações avaliadas apresentou sinais de instabilidade quando submetidas ao teste de centrifugação.

O teste de centrifugação indica, em curto espaço de tempo, possíveis instabilidades desencadeadas por processos físicos ou físico-químicos, que podem ocorrer no sistema. Este teste produz estresse na amostra simulando um aumento na força de gravidade, fazendo com que haja maior mobilidade das partículas e antecipando possíveis instabilidades que poderão ser observadas através de precipitação ou separação de fases (6).

De acordo com o guia de estabilidade da Anvisa (2004), para a execução do estudo de estabilidade preliminar, as amostras devem ser submetidas ao teste de centrifugação e não apresentar precipitação, separação de fases ou qualquer outra alteração ao final do teste (5). As 5 formulações avaliadas não apresentaram alterações quando submetidas ao teste de centrifugação, e da mesma forma, também não apresentaram alterações quando armazenadas a temperatura de $25^{\circ} \mathrm{C}$. Portanto pode-se inferir que a instabilidade observada nas formulações F1, F2 e F3 está provavelmente ligada a alterações fí- 
sico-químicas desencadeadas nas amostras pela ação da temperatura.

A Tabela 3 descreve a variação do $\mathrm{pH}$ das formulações nas temperaturas e nos períodos avaliados.

A avaliação do $\mathrm{pH}$ é um dos parâmetros utilizados para monitorar alterações na estrutura da formulação que nem sempre são perceptíveis visualmente. Esta análise físico-química pode indicar problemas de estabilidade, ou interação entre os componentes da formulação. Mudanças no valor do $\mathrm{pH}$ ao longo do tempo podem indicar a ocorrência de reações de hidrólise, oxidação, ou contaminação bacteriana $(5 ; 12)$.

Como pode ser observado na Tabela 3, houve uma diminuição significativa do valor do $\mathrm{pH}$ da formulação F4, nas distintas condições de temperatura. Essa diminuição pode ser explicada pela hidrólise dos triglicerídeos com formação de ácido graxos, que acidificam o meio, e quanto maior a quantidade de álcool cetoestea- rílico, maior será a formação de ácidos graxos e, portanto, menor será o valor de $\mathrm{pH}$. Masmoudi e cols (2005) afirmaram que a diminuição do $\mathrm{pH}$ pode representar uma oxidação da fase oleosa com formação de hidroperóxidos ou mesmo a hidrólise de triglicerídeos levando a formação de ácidos graxos (13).

Entretanto, nas outras formulações, o pH apresentou-se estável entre 5,5 - 6,5, não diferindo, significativamente, em relação ao tempo transcorrido e nas distintas temperaturas de acondicionamento. Em estudos desenvolvidos por Souza e Ferreira (2010) sobre a estabilidade de creme não-iônico com a utilização de álcool cetoestearílico etoxilado, foi observado que os valores de pH não apresentaram alterações marcantes (14). Fato muito importante, pois, segundo Melo e Siqueira (2012), um $\mathrm{pH}$ baixo pode modificar a localização dos elétrons na molécula dos filtros solares, e o produto passa a absorver radiação solar num comprimento de onda fora do desejado, o que prejudica a sua eficácia (15).

Tabela 3. Resultados da medição do pH das formulações analisadas durante o período de 15 dias. ( $n=3$; média \pm desvio padrão)

\begin{tabular}{|c|c|c|c|c|}
\hline \multicolumn{5}{|c|}{ pH } \\
\hline \multicolumn{5}{|c|}{ T.A } \\
\hline & \multicolumn{2}{|c|}{$\left(25^{\circ} \mathrm{C} \pm 2\right)$} & \multicolumn{2}{|c|}{$37^{\circ} \mathrm{C} \pm 2$} \\
\hline formulação & $1^{\circ} \mathrm{dia}$ & $15^{\circ}$ dia & $1^{\circ}$ dia & $15^{\circ}$ dia \\
\hline F1 & $6,10 \pm 0,04$ & $6,03 \pm 0,33$ & $6,10 \pm 0,07$ & $*$ \\
\hline F2 & $5,10 \pm 0,01$ & $4,99 \pm 0,24$ & $5,10 \pm 0,05$ & $*$ \\
\hline F3 & $5,68 \pm 0,09$ & $5,65 \pm 0,34$ & $5,68 \pm 0,21$ & $*$ \\
\hline F4 & $6,62 \pm 0,11$ & $5,72 \pm 0,41$ & $6,62 \pm 0,1$ & $5,44 \pm 0,28$ \\
\hline F5 & $5,50 \pm 0,01$ & $5,24 \pm 0,15$ & $5,50 \pm 0,05$ & $4,82 \pm 0,45$ \\
\hline
\end{tabular}

* Presença de instabilidade na formulação.

Foi observado, ainda, neste estudo, que as amostras acondicionadas a $37^{\circ} \mathrm{C}$, apresentaram uma maior redução no valor do pH, em relação as formulações mantidas a temperatura ambiente $\left(25^{\circ} \mathrm{C}\right)$. Estes resultados confirmam a hipótese de que altas temperatura desencadeiam reações físico-químicas no sistema, e estas reações, dependendo da composição quali e quantitativa da emulsão, pode levar a processos de instabilidade.

A Tabela 4 apresenta a viscosidade das formulações F1, F2, F3, e F5 acondicionadas à temperatura ambiente $\left(25 \pm 2^{\circ} \mathrm{C}\right)$ e a $37 \pm 2^{\circ} \mathrm{C}$ durante o período de 15 dias.

A determinação da viscosidade permite avaliar se a preparação apresenta a consistência ou fluidez apropriadas, e fornece informações sobre o comportamento do produto ao longo do tempo (5). Segundo Lachman e cols (2001) a viscosidade de uma emulsão pode ser alterada pela composição de lipídios, pela proporção entre fase aquosa e oleosa, pela concentração de doadores de viscosidade e emulsionantes, assim como pela presença de polímeros (16). Como observado na Tabela 4, as formulações analisadas apresentaram valores de viscosidade distintos, e os mesmos foram decaindo ao longo do tempo, tanto para as amostras analisadas a temperatura ambiente $\left(25 \pm 2^{\circ} \mathrm{C}\right)$, quanto à $37 \pm 2^{\circ} \mathrm{C}$. De acordo com Woolfson e colaboradores (2000), este fato pode indicar alterações do agente de viscosidade presente nas formulações ao longo do tempo (18). Para as formulações F1, F2 e F3 foi utilizado o cosmowax J como agente de viscosidade, já nas formulações F4 e F5 o agente utilizado foi o álcool cetoestearílico. Nas formulações F1, F2 e 
F3 há uma menor quantidade de álcool cetoestearílico quando comparado à F4 e F5, esse fato permite sugerir que quanto maior quantidade de álcool cetoestearílico na composição, maior será a viscosidade do produto. Outro dado, que corrobora esta hipótese é a alta viscosidade apresentada pela formulação $\mathrm{F} 4$, que é, dentre todas, a formulação com maior concentração de álcool cetoestearílico. Não foi possível mensurar experimentalmente a viscosidade da formulação $\mathrm{F}$, pois a mesma se apresentou muito espessa, de modo a impossibilitar a utilização do spindle L3, que foi o spindle padrão adotado no ensaio.

Tabela 4. Resultados da análise de viscosidade das formulações durante o período de 15 dias. ( $n=3$; média \pm desvio padrão)

\begin{tabular}{|c|c|c|c|c|}
\hline \multicolumn{5}{|c|}{ Viscosidade (cP) } \\
\hline \multicolumn{7}{|c|}{ T.A } \\
\hline Formulação & $\mathbf{1}^{\circ}$ dia & $\mathbf{2 5}^{\circ} \mathbf{C} \pm \mathbf{2}$ & \multicolumn{2}{c|}{$\mathbf{3 7}^{\circ} \mathbf{C} \pm \mathbf{2}$} \\
\hline F1 & $12000 \pm 0,015$ & $1057 \pm 0,18$ & $12000 \pm 0,41$ & $\mathbf{1 5}^{\circ}$ dia \\
\hline F2 & $20154 \pm 0,2$ & $1210 \pm 0,35$ & $20154 \pm 0,12$ & $*$ \\
\hline F3 & $40105 \pm 0,45$ & $24860 \pm 0,25$ & $41340 \pm 0,15$ & $*$ \\
\hline F4 & ND & ND & ND & ND \\
\hline F5 & $41340 \pm 0,34$ & $15170 \pm 0,11$ & $40105 \pm 0,07$ & $29040 \pm 0,27$ \\
\hline
\end{tabular}

* Presença de instabilidade nas formulações;

CP - centipoise; ND - Não determinado

Milan e cols (2007) verificaram que emulsões cosméticas não-iônicas utilizando o álcool cetoestearílico, como agente espessante, demonstraram maior viscosidade e melhor fluidez ao longo do tempo, quando comparada a emulsões que utilizavam a hidroxietilcelulose como agente espessante (17). O decréscimo no valor da viscosidade após 15 dias para todas as preparações ocorreu, possivelmente, devido a processos de organização estrutural do sistema não iônico, pois na ausência de cargas do tensoativo, os glóbulos da emulsão tendem a levar mais tempo para se estabilizarem.

Outra observação relevante neste estudo foi o fato da formulação F3, que difere da F2 apenas por não possuir em sua composição dióxido de titânio, apresentar viscosidade muito superior à formulação F2. Isso sugere que a viscosidade está mais relacionada com o equilíbrio entre os componentes do sistema, do que com a quantidade de agente de viscosidade na preparação.

O conhecimento da viscosidade aparente do sistema, ainda que informativo, é fundamental do ponto de vista farmacotécnico, pois o comportamento reológico adequado é exigido para que a atividade terapêutica, ou as funções cosméticas do produto, sejam asseguradas (18).

A determinação do fator de proteção solar (FPS) foi realizada apenas para as formulações F1 e F5.
O teste foi conduzido após o termino do estudo de estabilidade preliminar, com as amostras que foram armazenadas a temperatura ambiente $\left(25 \pm 2^{\circ} \mathrm{C}\right)$. F1 foi selecionada por representar uma formulação comercial que estava apresentando problemas de estabilidade, e F5 por se mostrar a preparação com as melhores características durante o desenvolvimento deste estudo, e desta forma ser a nova proposta de formulação para substituir F1.

Foram obtidos os valores de FPS 22,97 $\pm 0,08$ e FPS $24,32 \pm 0,025$ para F1 e F5, respectivamente. Os resultados mostram que F5 apresentou valor de FPS mais elevado em relação a F1. O valor do FPS das formulações desenvolvidas neste estudo é referente à associação dos filtros orgânicos benzofenona-3 (benzophenone-3), que confere proteção UVA e UVB; salicilato de octila (ethylhexyl salicylate) que oferece proteção UVB; e metoxicinameto de octila (ethylhexyl methoxycinnamate) que confere proteção UVB. Como pode ser observado na Tabela 1, a formulação $\mathrm{F} 1$ possui maiores quantidades dos filtros benzofenona-3 e metoxicinamato de octila, e menor quantidade de salicilato de octila, em relação à formulação F5. Esta alteração na proporção de filtros solares orgânicos foi realizada no desenvolvimento da formulação com o intuito de contribuir para uma melhor estabilidade e segurança da formulação final, tendo em vista que a benzofenona-3, é um componente com potencial alergênico, além de ser um filtro de difícil so- 
lubilização (2), o que pode acarretar processos de instabilidade na formulação final. Por outro lado o salicilato de octila é um composto estável, seguro e que age como solvente para filtros solares fracamente solúveis como é o caso das benzofenonas (2). Portanto diminuir a quantidade de benzofenona-3 e aumentar a quantidade de salicilato de octila na formulação se mostrou uma alternativa adequada, que resultou em aumento do FPS da formulação. Este aumento no valor do FPS da formulação F5 em relação a F1 pode ser explicado, tanto, pela maior quantidade de salicilato de octila na composição, o que favorece a uma maior captação dos raios UVB, quanto pelo aumento da quantidade deste filtro promover a solubilização dos demais filtros orgânicos, e desta forma potencializar a ação fotoprotetora da formulação final. Os protetores solares são utilizados com o intuito de prevenir temporariamente os danos causados pela radiação solar (19), desta forma o método desenvolvido por Mansur e cols (1986) é um método rápido e eficaz para avaliar o FPS de substâncias e produtos (7), sendo empregado na fase de desenvolvimento de formulações e como ensaio de rotina no controle de qualidade, pois apresenta adequada correlação com o método in vivo.

\section{REFERÊNCIAS}

1. Guaratini T; Callejon DR; Pires DC; Lopes JNC. Fotoprotetores derivados de produtos naturais: perspectivas de mercado e interações entre o setor produtivo e centros de pesquisa. Rev. Quím. Nov. 2009; 32(3):717-721. DOI: 10.1590/S0100-40422009000300015

2. Balogh TS; Velasco MVR; Pedriali CA; Kaneko T M; Baby AR. Proteção à radiação ultravioleta: recursos disponíveis na atualidade em fotoproteção. An. Bras. Dermatol. 2011; 86:732-742. DOI: 10.1590/S0365-05962011000400016

3. Franquilino E. Proteção solar. Formulação e avaliação. Produtos seguros e eficazes. Cosmetics \& Toiletries. 2014; (26):15-16.

4. Lima CG; Vilela AFG; Silva AAS; Piannovski AR; Silva KK; Carvalho VFM; Musis CR; Machado SRP; Ferrari M. Desenvolvimento e avaliação da estabilidade física de emulsões O/A contendo óleo de babaçu (Orbignya oleifera). Rev. Bras. Farm. 2008; 89: 239-245.

5. ANVISA. Agência Nacional de Vigilância Sanitária Guia de estabilidade de produtos cosméticos. Brasília, 2004. v. 1.

6. Larentes IA. Avaliação preliminar da estabilidade de cremes fotoprotetores de farmácias magistrais [TCC]. Maringá-PR: Faculdade Integrada da Grande Fortaleza (FGF); 2009.

\section{CONCLUSÃO}

A eficácia do fotoprotetor não depende apenas dos tipos e quantidades de filtros solares envolvidos, mas das características químicas e físicas de todos os seus componentes. As formulações F4 e F5, desenvolvidas com álcool cetoestearílico associado ao álcool cetoestearílico etoxilado, mostraram estabilidade, mesmo quando submetidas a temperatura elevada $\left(37^{\circ} \mathrm{C} \pm 2\right)$. Quanto maior a quantidade de álcool cetoestearílico, maior será a viscosidade do produto e mais acidificada ficará a amostra. A prédispersão de dióxido de titânio foi responsável pelo aumento da fluidez da amostra F2. A formulação F5 foi aprovada no teste de estabilidade preliminar e apresentou viscosidade adequada, portanto será produzida em escala piloto para realizar os testes de estabilidade acelerada e de longa duração.

\section{AGRADECIMENTOS}

À Farmácia Escola da Universidade Federal do Ceará pelo financiamento do projeto.

7. Mansur JS; Breder MNR; Mansur MCA; Azulay RD. Determinação do Fator de Proteção Solar por espectrofotometria. An. Bras. Dermatol. 1986; 61(3):121-124.

8. Oliveira AZM. Desenvolvimento de formulações cosméticas com ácido hialurônico [Dissertação]. Portugal: Universidade do Porto: Programa de Pós Graduação em Tecnologia Farmacêutica; 2009.

9. Veríssimo LM. Desenvolvimento de sistemas farmacêuticos emulsionados para veiculação galênica [Dissertação]. Natal: Universidade Federal do Rio Grande do Norte: Programa de Pós Graduação em Genética e Biologia Molecular; 2007.

10. Zanon AB. Aspectos teóricos e práticos sobre a avaliação da estabilidade de emulsões manipuladas em farmácia [TCC]. Porto Alegre: Universidade Federal do Rio Grande do Sul; 2010.

11. Tolotti MF. Protetores solares à base de filtros inorgânicos: desenvolvimento e caracterização. [Dissertação]. Guarapuava: Universidade Estadual do Centro Oeste; 2012.

12. Aulton ME. Delineamento de Formas Farmacêuticas. 2. Ed. Porto Alegre: Artmed, 2005.

13. Masmoudi H; DréauYL; Piccerelle P; Kister J. The evaluation of cosmetic and pharmaceutical emulsions 
aging process using classical techniques and a new method: FTIR. Int. J. Pharm. 2005; 289(1):117-131. DOI: 10.1016/j.ijpharm.2004.10.020

14. Souza VB; Ferreira JRN. Desenvolvimento e estudos de estabilidade de cremes e géis contendo sementes e extratos do bagaço da uva Isabel (Vitis labrusca L.). Rev. de Ciênc. Farm. Bras. Aplic. 2010; 31(3): 217-222.

15. Melo MR; Siqueira APNDF. Desenvolvimento de uma loção fotoprotetora, avaliação da estabilidade e determinação in vitro do FPS. Perquirere, Patos de Minas, MG, 2012; 9(1):81-97.

16. Lachman L; Lieberman HA; Kanig JL. Teoria e Prática na Indústria Farmacêutica. Lisboa: Fundação Calouste Gulbenkian, 2001.
17. Milan ALK; Milão D; Souto AA; Corte TWF. Estudo da hidratação da pele por emulsões cosméticas para xerose e sua estabilidade por reologia. Rev. Bras. Ciênc. Farm. Porto Alegre. 2007; 43(4): 649-657.

18. Woolfson AD; Malcolm RK; Campbell K; Jones DS; Russell JA. Rheological, mechanical and membrane penetration properties of novel dual drug systems for percutaneous delivery. J. Control. Release. 2000; 67(2):395408. DOI: 10.1016/S0168-3659(00)00230-3

19. Barbosa JS; Louchard BO; Araújo TG. Fatores Determinantes para a Eficácia de Filtros Solares Inorgânicos Cosmetics \& Toiletries Brasil, 2017; 29(1): 64-66. 